\title{
Improvement of radiotracers experiments in mass transfer processes
}

\author{
Leszek Petryka ${ }^{1, a}$, Volodymyr Mosorov ${ }^{2}$, Marcin Zych ${ }^{1}$, Robert Hanus ${ }^{3}$, Jerzy Sobota $^{4}$, Marek Jaszczur ${ }^{1}$, and Dariusz \\ Świsulski ${ }^{5}$ \\ ${ }^{1}$ AGH University of Science and Technology, Kraków 30-059, Poland \\ ${ }^{2}$ Łodz University of Technology, Łodz 90-924, Poland \\ ${ }^{3}$ Rzeszów University of Technology, Rzeszów 35-959, Poland \\ ${ }^{4}$ Wrocław University of Environmental and Life Sciences, Wrocław 50-375, Poland \\ ${ }^{5}$ Gdańsk University of Technology, 80-233 Gdańsk, Poland
}

\begin{abstract}
The greatest part of industrial mass transfer is based on continuous transportation of different substances throughout especially constructed installations. In that kind of practice, a flow of such mass in each part determines the process unit and can be described by the residence time distribution (RTD) of the substance inside this unit. This parameter may be evaluated by the properly arranged tracer experiment. It is worthwhile to notice that flow components are labeling for a sufficiently long time for offering a lot of advanced techniques fitted to the particular circumstances.
\end{abstract}

\section{Introduction}

An analysis of a selected particle or a component movement in any flow is possible using tracers. In this approach to investigation, especially convenient are radioactive elements induced or placed in the examined substance and consequently detected by a probe located outside of the process $[1,2]$. That opportunity ensures radioisotopes emitting gamma radiation, when energy of photons exceeds a hundred $\mathrm{kVe}$. The above mentioned simple idea has been applied for almost a hundred years and presently may offer many additional improvements [3].

The paper focused on a case of the hydraulic transportation of a solid phase in pipes and in such processes as a mineral industry where descriptions of the mass transfer by a residence time or velocity distribution are applied [4]

\section{Velocity Measurements}

A popular example of radiotracer application is solid particles transportation by liquid through a pipeline, presented in Fig. 1. When we imagine that a single labelled particle is introduce to the cross section $S_{0}$ with the axial momentum equal to zero. Consequently during transportation through initial section $L_{l}$, provided the same axial velocity of that grain, as the most in the vicinity. In practice, the $L_{1}$ distance may vary from several centimeters to meters, depending on accuracy demanded and turbulence of the flow $[5,6]$.

\footnotetext{
${ }^{\mathrm{a}}$ Corresponding author: Leszek.Petryka@fis.agh.edu.pl
}

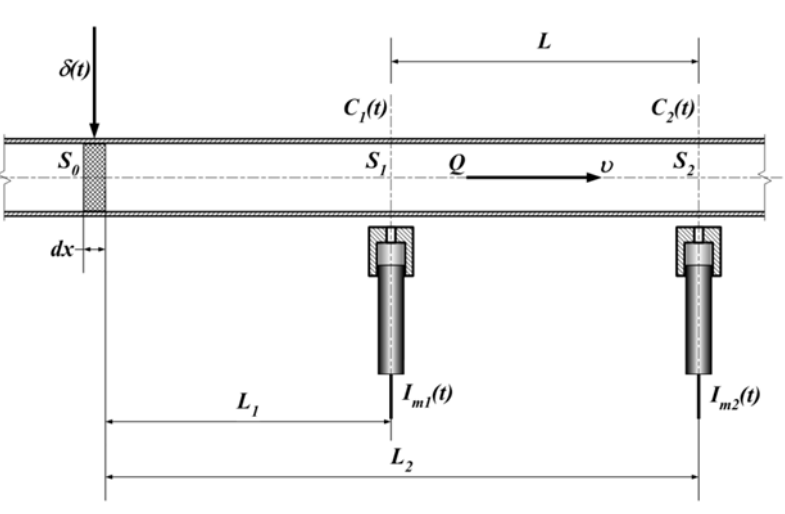

Fig. 1. Radiotracer measurement in a pipe: $S_{0}$ - tracer injection section, $S_{1}-$ beginning of measuring section, $S_{2}-$ end of measuring section, $C(t)$ - tracer concentration, $Q$ - volumetric flow rate, $I_{m}-$ measured count rate.

After fulfilling that condition, the residence time $t$ in the pipe section between $S_{1}$ and $S_{2}$ allows determining the local axial velocity $v_{L}$ of the marked grain representing the solid phase, according to:

$$
v_{L}=L / t
$$

In case of the one particle experiment there is no problem with determination of time in which a tracer is passing by both the $S_{1}$ and $S_{2}$ cross sections of the pipe, so the residence time:

$$
t=t_{2}-t_{1}
$$

The experiment described above would be repeated several times, for the mean velocity $v_{m}$ of entire labelled phase determination. That method is comparatively 
simple, but long lasting and during all the time the same stable condition of the flow is demanded

$$
v_{m}=\frac{1}{n} \sum_{i=1}^{n} v_{i}
$$

Huge reduction of the time spent for the velocity measurement may be achieved by collecting significant number of marked particles in the single portion injected to $S_{0}$ section in a manner resembling Dirac's $\delta(t)$ distribution. Nevertheless, those facilities are covered by more difficult measurement and smaller accuracy of results $[7,8]$.

In such cases it should be assumed that:

$$
C_{\text {in }}(t)=\delta(t)
$$

is uniformly distributed in a total cross-section of a stream.

Additionally during this kind of velocity measurement, the activity of tracers in the injected portion should be reduced to a level accepted by the applied probes and safe for the staff involved in the investigation.

Moreover, the injected sample should be statistically significant but still below the level devastating the process stability.

Furthermore, in this method, the recorded data processing is more complicated by the background determinations and residence time evaluation procedures. In consequence a few groups in the world are sufficiently experienced for the proper arrangement of such measurements.

\section{Single particle's velocity measurement}

Fig. 2 shows the measurement part of the hydraulic installation build up in the Central Mining Institute in Katowice, Poland.

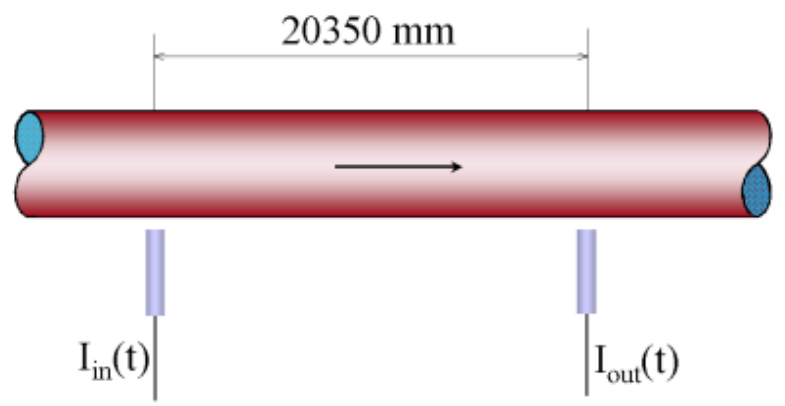

Fig. 2. The idea of the transit time measurement.

During experiments testing solid particles' velocity determination, the sandstone grains, about $10 \mathrm{~mm}$ diameter, were transported by water through a steel pipe over $40 \mathrm{~m}$ long, and of $185 \mathrm{~mm}$ inner diameter. The installation used for the testing is presented in Fig. 3, where the rotation pump forced the flow containing a mixture of almost equal sandstone grains and water with $1.2 \mathrm{Mg} / \mathrm{m}^{3}$ density. In that case, the solid phase was represented by a single grain containing radioactive isotopes of ${ }^{24} \mathrm{Na}$ and ${ }^{46} \mathrm{Sc}$. Due to that, probes 1 and 2 provided "yes" or "no" information only.

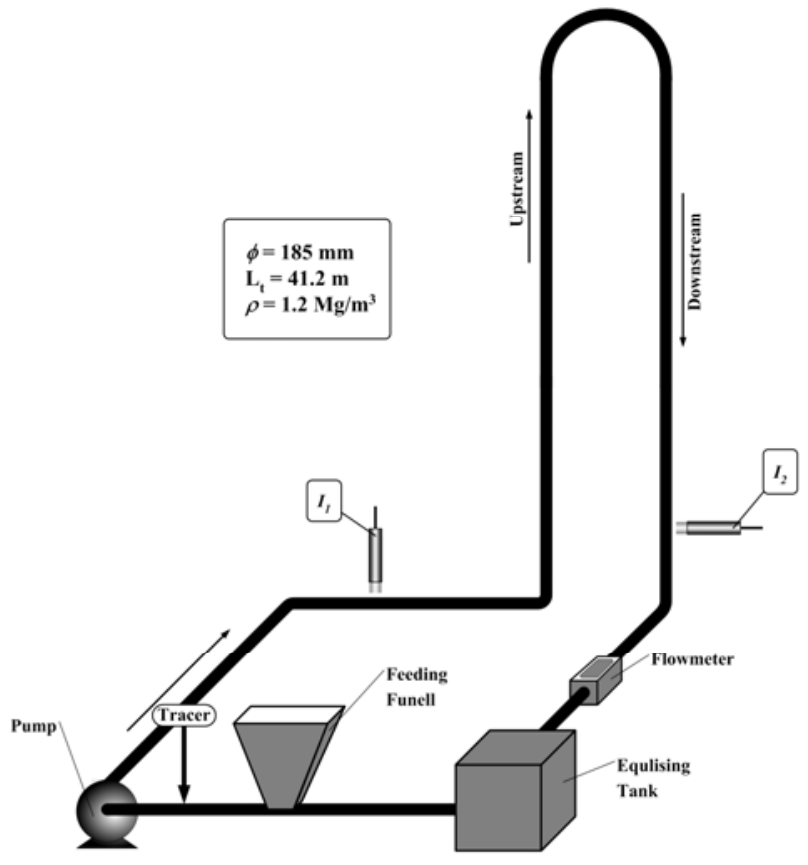

Fig. 3. The hydraulic installation used for the velocity measurement's testing.

This means that the data acquisition set is responding "no" almost all of the time, except of the situations where the labeled particle is just in the particular $S_{i}$ crosssection. Such binary information is practically not influenced by the background radiation. Moreover, due to the mixture recirculation in the closed loop, the recorded data, allowed at least 30 times to observe the labelled grain passing through the measuring section. Due to that, the set of fluctuated residence times, shown in Fig. 4 was recorded. Their histogram was very similar to the residence time distribution $E_{12}(t)$ presented in Fig. 5.

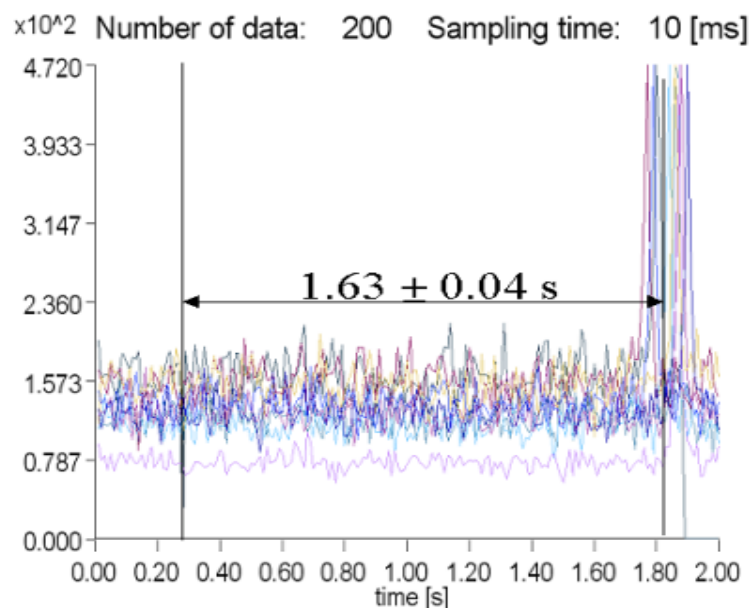

Fig. 4. The results of the single particle's transit time measurements.

\section{Traditional solid particle's velocity measurement}

The mostly applied radiotracer's velocity measurement based on the join injection of the sample, which in our 
case consisted of 30 grains in roughly $10 \mathrm{~mm}$, labelled by the radioactive isotopes. Moreover, in that case, also the probes 1 and 2 may record recirculation of total radioactive sample.

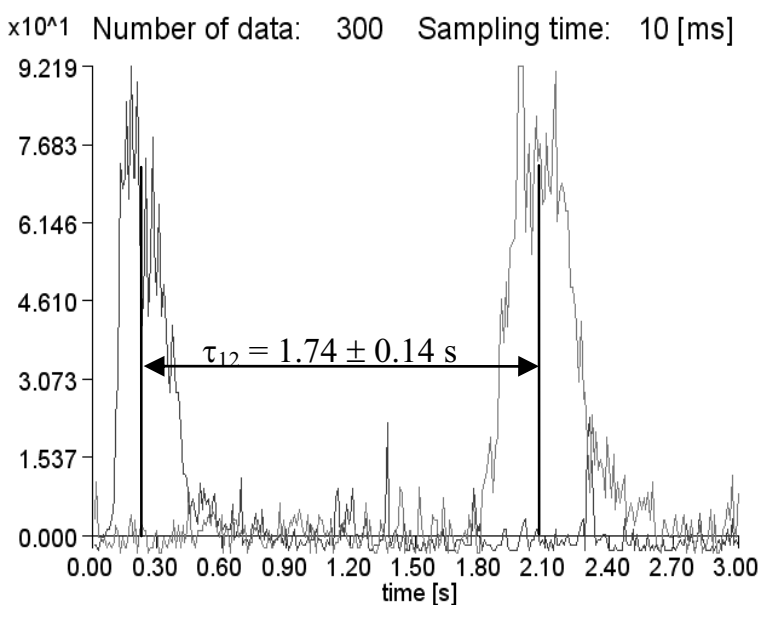

Fig. 5. The first peaks recorded by probes 1 and 2 in the measurement LA4003.

It is worth to notice that the ideal injection of the tracer in the examined flow should have a form of $\delta(t)$ (Dirac function of time, but uniformly distributed in total cross-section of a stream). In practice, this condition of injection is difficult to fulfill, nevertheless it is readily applied due to limited amount of the tracer activity and quick measurement. Often the assumption of impulse injection is satisfied when the tracer is introduced in short time in relation to mean axial velocity and much shorter than its residence time distribution $E_{l}(t)$ in the mixing distance $L_{l}$.

Though as yet, in practice it is hard to guarantee uniform distribution of the tracer in the cross-section of the stream, but the same effect may be achieved by assuring sufficiently long $L_{i}$ distance which should allow even distribution of the tracer and give the same velocity as for the surrounding particles.

In the presented investigation, the above conditions were fulfilled with the use of radiotracers' injection into the inlet of the pump. Subsequently in the result of proper selection of tracer and measurement's geometry, the linear relation between tracer concentration in the $i$ crosssection $C_{i}(t)$ and count rate originated from the tracer $I_{i}(t)$ may be obtained:

$$
C_{i}(t)=D I_{i}(t)
$$

and for the $i$ probe

$$
I_{i}(t)=I_{m i}(t)-I_{B i}
$$

where:

$D_{i} \quad$ - calibration coefficient,

$I_{i}(t)$ - effective counts rate originated by the tracer,

$I_{B i}$ - count rate representing the background radiation.

The classical tracer velocity measurement mostly applies the moment's method. The mean axial velocity in this way may be calculated according to Danckwerts by the residence time distributions of tracer derived from the following equations [9]:

$$
E_{i}(t)=\frac{C_{i}(t)}{\int_{0}^{\infty} C_{i}(t) d t}=\frac{I_{i}(t)}{\int_{0}^{\infty} I_{i}(t) d t}
$$

and the ordinary $j$ moment of the above distribution

$$
M_{i j}=\int_{0}^{T} t^{j} E_{i}(t) d t
$$

where $T$ - time of observation.

In practice, the $T$ should last until significant part of the tracer will leave the process, so when in consequence the radiation emitted by the tracer may be neglected.

For these calculations, the most important is the first moment, representing mean residence time of the tracer in the mixing section of the stream $L_{l}$ :

$$
\tau_{i}=\int_{0}^{T} t E_{i}(t) d t
$$

Similarly $\tau_{2}$ may be calculated for the section $L_{2}$ and then mean transportation delay:

$$
\tau_{12}=\tau_{2}-\tau_{1}
$$

according to symbols presented in Fig. 1,

$$
v=L / \tau_{12}
$$

and the central $j$ moment of $E_{i}(t)$ :

$$
\mu_{j i}=\int_{0}^{T}\left(t-\tau_{i}\right)^{j} E_{i}(t) d t
$$

The velocities uncertainty $u\left(v_{i}\right)$ may be approximated by $\sigma_{i}$ derived from the second central moment:

$$
\sigma_{i}^{2}=\mu_{2 i}=\int_{0}^{T}\left(t-\tau_{i}\right)^{2} E_{i}(t) d t
$$

In consequence, the uncertainty of the transportation delay, according to Petryka and Keblowski [10]:

$$
u\left(\tau_{12}\right)=\sqrt{\left(\Delta \tau_{1}\right)^{2}+\left(\Delta \tau_{2}\right)^{2}}
$$

for the data delivered by the first probe, when low indexes denote to the moments order:

$$
\Delta \tau_{1}=\sqrt{\left(\frac{\Delta M_{1}}{M_{0}}\right)^{2}+\left(\frac{\Delta M_{0}}{M_{0}^{2}} M_{1}\right)^{2}}
$$


where

$$
\Delta M_{1}=\sqrt{M_{2}+\left[\frac{1}{2} \Delta t n(n+1) \sigma_{B}\right]^{2}}
$$

and

$$
\begin{gathered}
\Delta M_{0}=\sqrt{M_{0}+\left(n \sigma_{B}\right)^{2}} \\
\sigma_{B}=\sqrt{\frac{\sum_{i=1}^{n}\left(I_{B i}-I_{B}\right)^{2}}{n(n-1)}}=\sqrt{\frac{\sum_{i=1}^{n} I_{B i}^{2}-n I_{B}^{2}}{n(n-1)}} \\
I_{B}=M_{B 0} / n
\end{gathered}
$$

$n$ - total number of the background data,

$M_{B O}$ - zero moment of the background.

In the same way $u\left(\tau_{2}\right)$ may be calculated, based on data delivered by the second probe.

The example illustrating the above calculations was selected from the measurements conducted in the Main Mining Institute Laboratory in Laziska, Poland. The schema of this installation is presented in Fig. 3. During applied measurement the flow consisted of sandstones transported by water. As the tracer was selected a sample consisting of 30 sandstone grains in roughly $10 \mathrm{~mm}$, activated in the Nuclear Reactor, so radiation of this marker was emitted mostly by ${ }^{24} \mathrm{Na}$ and ${ }^{46} \mathrm{Sc}$. The established distance between probes $L=20.350 \mathrm{~m}$, provided transportation delay $\tau_{12}=1.74 \pm 0.14 \mathrm{~s}$, and mean velocity $v_{12}=11.6 \pm 0.9 \mathrm{~m} / \mathrm{s}$. The data recorded during that experiment is shown in Fig. 5, and deliver results illustrating the described method according to Petryka and Keblowski [ibid.]. An example of $I_{i}(\mathrm{t})$ distributions obtained in radiotracer measurement in Laziska Laboratory, is shown in Fig. 6 [ibid.].

\section{Deconvolution calculations}

In the deconvolution method, the radiation recorded by the first probe $I_{m I}(\mathrm{t})$ may by assumed as the input tracer to the pipes' measuring section $L$.

Actual radiotracer investigations provided signals $I_{m l}(\mathrm{t})$ and $I_{m 2}(\mathrm{t})$ originated by the tracer but also containing noise. Some part of that noise is caused by measuring equipment and electronics. Completed measurements showed that the noise level in recorded signals may be significantly reduced by an improvement of the measuring geometry and proper sampling time selection, Petryka [11].

Moreover, the deconvolution method is sensitive mostly to the high frequency disturbances, so its influence may be reduced by cutoff frequencies introduced to both $I_{m i}(\mathrm{t})$ signals. These filtrations give $I_{m l}{ }^{\prime}(\mathrm{t})$ and $I_{m 2}(\mathrm{t})$ signals, according to Proakis and Manolakis [12]. Result of this procedure application to the La4003 measurement is presented in Fig. 7 and Table 1.

According to that procedure, the residence time distribution $E_{12}(\mathrm{t})$ may be derived from the Duhamel integral:

$$
\begin{aligned}
& I_{m 2}{ }^{\prime}(t)=\frac{1}{A_{f}} \int_{0}^{t} I_{m 1}{ }^{\prime}(t-\xi) E_{12}(\xi) d \xi= \\
& =I_{m 1}{ }^{\prime}(t) * E_{12}(t)
\end{aligned}
$$

where:

$A_{f}$ - normalizing coefficient,

$I_{m l}{ }^{\prime}(\mathrm{t})$ and $I_{m 2}{ }^{\prime}(\mathrm{t})$ - signals representing tracer, prepared for convolution calculations.

In practice, the above integral might be solved with help of the Fourier transform.
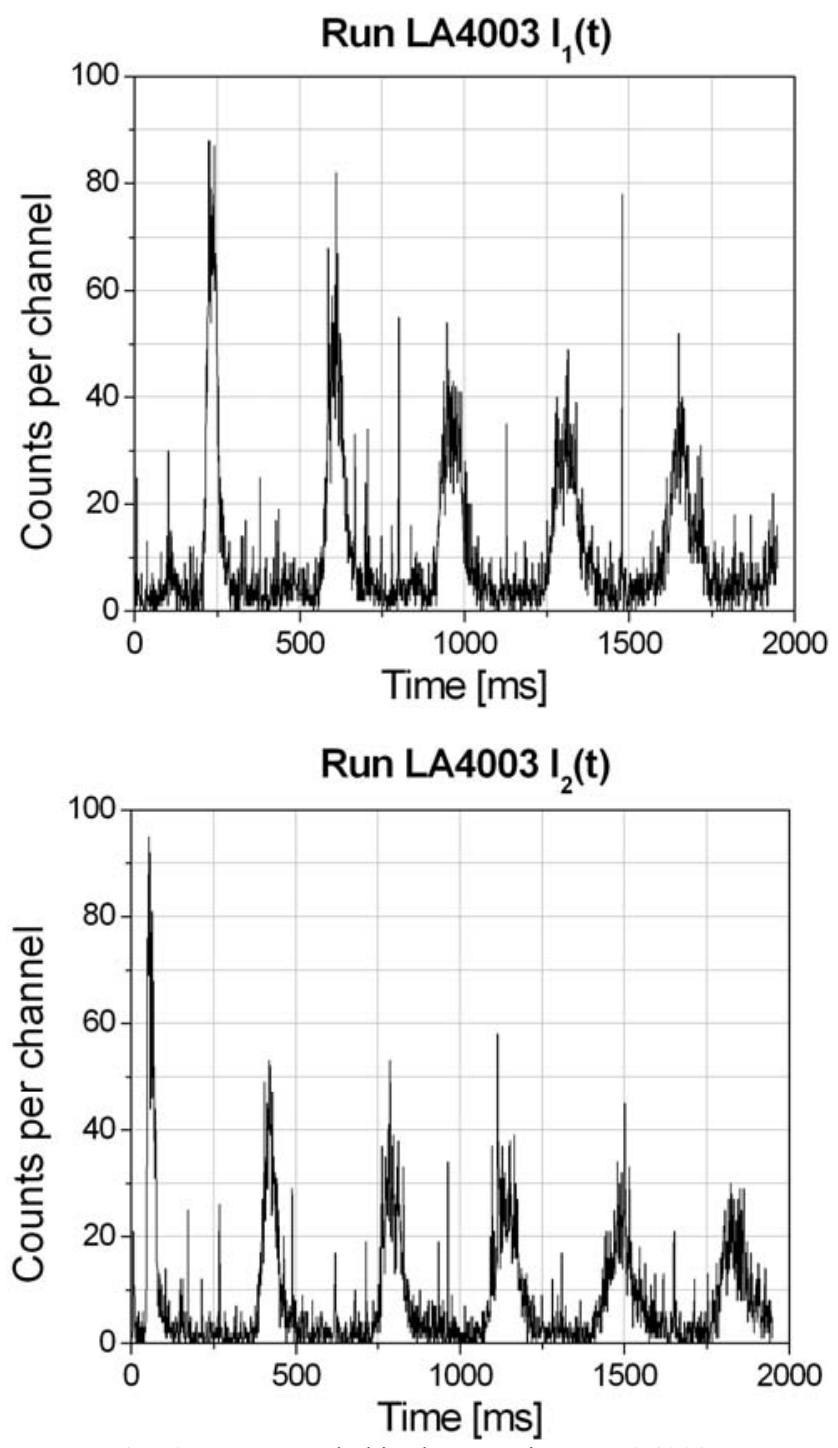

Fig. 6. Data recorded in the experiment LA4003.

For signal from the first probe:

$$
I_{m 1}{ }^{\prime}(\omega)=\int_{0}^{\infty} I_{m 1}{ }^{\prime}(t) e^{-i \omega t} d t
$$

Similarly, the signal from the second probe may be transformed and the residence time distribution in the 
frequency domain may be obtained from the following equation:

$$
E_{12}(\omega)=A_{f} \frac{I_{m 2}{ }^{\prime}(\omega)}{I_{m 1}{ }^{\prime}(\omega)}
$$

and at last:

$$
E_{12}(t)=\frac{1}{2 \pi i} \int_{-\infty}^{\infty} E_{12}(\omega) e^{i \omega t} d \omega
$$

Distribution obtained in this way may be directly applied to (9) for the mean residence time $\tau_{12}$ evaluation, spent for transportation of tracer through the distance $L$.

As a result, the mean velocity may be obtained from (11) and interpreted in the same way as one described in the following chapter.

\section{Cross-correlation analysis}

The Cross-correlation method recalls directly to the "single particle experiment" when the same, small statistical fluctuations of the tracer concentration $C_{I}(\mathrm{t})$, may be found in the $C_{2}(\mathrm{t})$ distribution after a delay necessary for the tracer transportation. The properly established measurement provides $I_{m l}(\mathrm{t})$ and $I_{m 2}(\mathrm{t})$ signals related to both concentrations by (5) and (6). After removing low frequency part and high frequency disturbances, it is possible to receive $I_{m 1}$,' $(\mathrm{t})$ and $I_{m 2}$,' $(\mathrm{t})$ signals which would be ergodic and still related to the small statistical fluctuations of the tracer concentration, as it was proposed by Petryka and Oszajec [13].

For sufficiently long observations $T$, the time $\tau$ spent for transportation may be observed by the following cross-correlation distribution:

$$
\begin{aligned}
& R_{12}(\tau)=\frac{1}{T} \int_{0}^{T} I_{m 1} "(t) I_{m 2} "(t-\tau) d t= \\
& =I_{m 1} "(t) \otimes I_{m 2} "(t)
\end{aligned}
$$

The above interpretation of the cross-correlation distribution suggests similarities between both $R_{12}(\mathrm{t})$ and $E_{12}(\mathrm{t})$ distributions, as it was proposed by Petryka [14].

Due to that, evaluation of the mean residence time $\tau_{12}$ in this method may be calculated from an equation similar to (7) and consequently the mean velocity may be obtained from (9) and compared to the previous methods. Described above procedures were tested in a numerous radiotracer experiments and one of them is accessible in the following chapter.

\section{Example of velocity measurement}

Distributions $I_{m I}(\mathrm{t})$ and $I_{m 2}(\mathrm{t})$ recorded during experiment LA4003 are given in Fig. 6. In both parts of that figure it is easy to recognize the recirculation of the marked sand sample, and find that the maximal count rate reaches almost $10000 \mathrm{cps}$ where sampling time was established to $10 \mathrm{~ms}$.
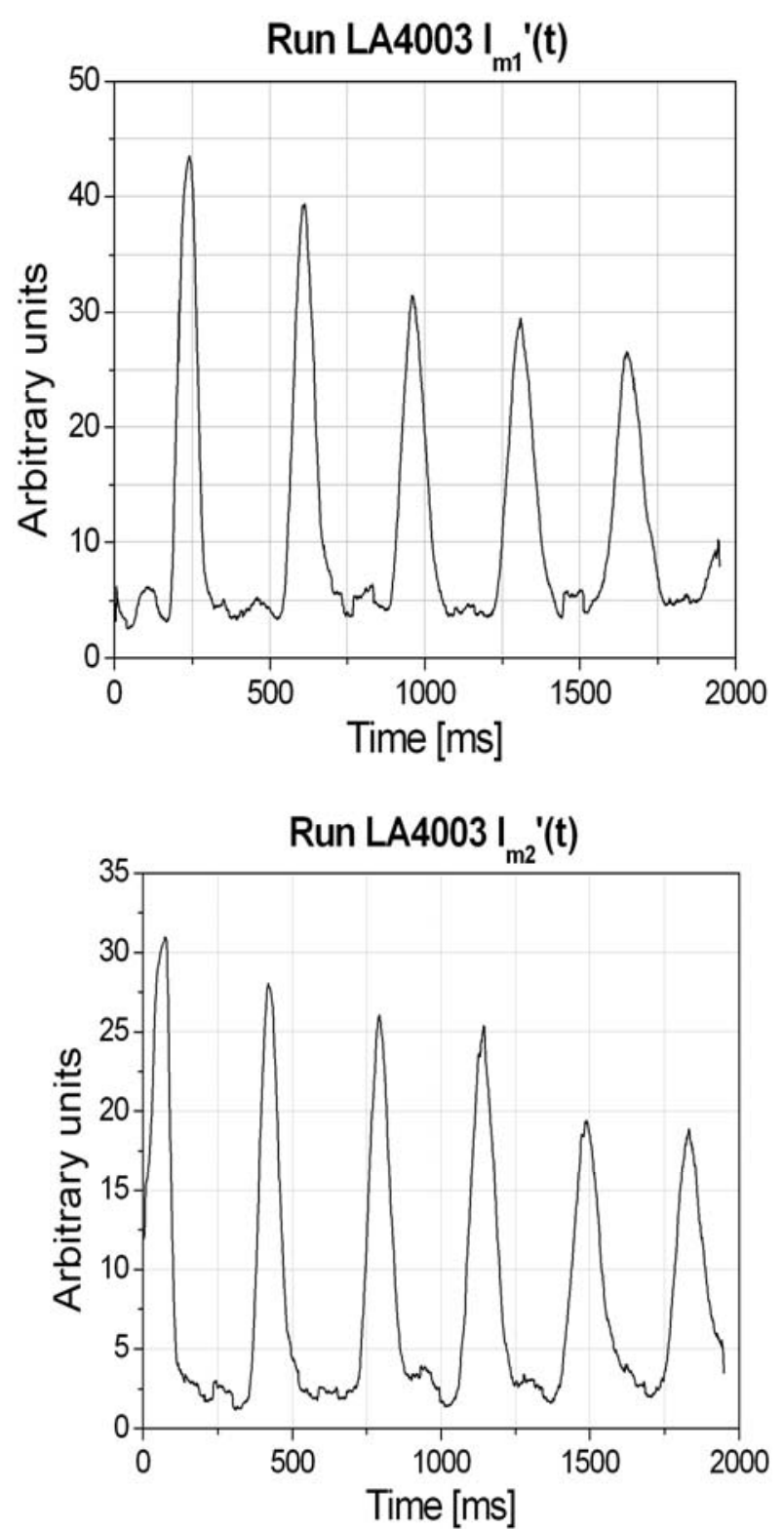

Fig. 7. Signals prepared for deconvolution calculations in the experiment LA4003.

In result of the Fourier transform according to (19) and after elimination of high frequencies above 65 internal units, both distributions $I_{m 1}{ }^{\prime}(\mathrm{t})$ and $I_{m 2}{ }^{\prime}(\mathrm{t})$, shown in Fig. 7 were obtained. Consequently, by deconvolution calculations of these data according to (18) up to (21), the residence time distribution $E_{12}(\mathrm{t})$ was derived.

Similarly, signals for correlation analysis were prepared. In this case, both low frequencies below 9 units and high one above 81 units were removed, producing signals $I_{m 1}, '(\mathrm{t})$ and $I_{m 2}, '(\mathrm{t})$ shown in Fig. 8.

Results of both approaches give very similar distributions shown in Fig. 9. Their processing by (9) and (11) provided results collected in Table 1. The uncertainty of transportation delay in case of the last two methods was evaluated as half of the selected sampling time. Consequently the relative uncertainty was obtained from the following equation:

$$
\delta=\frac{u\left(\tau_{12}\right)}{\tau_{12}} \cdot 100 \%
$$



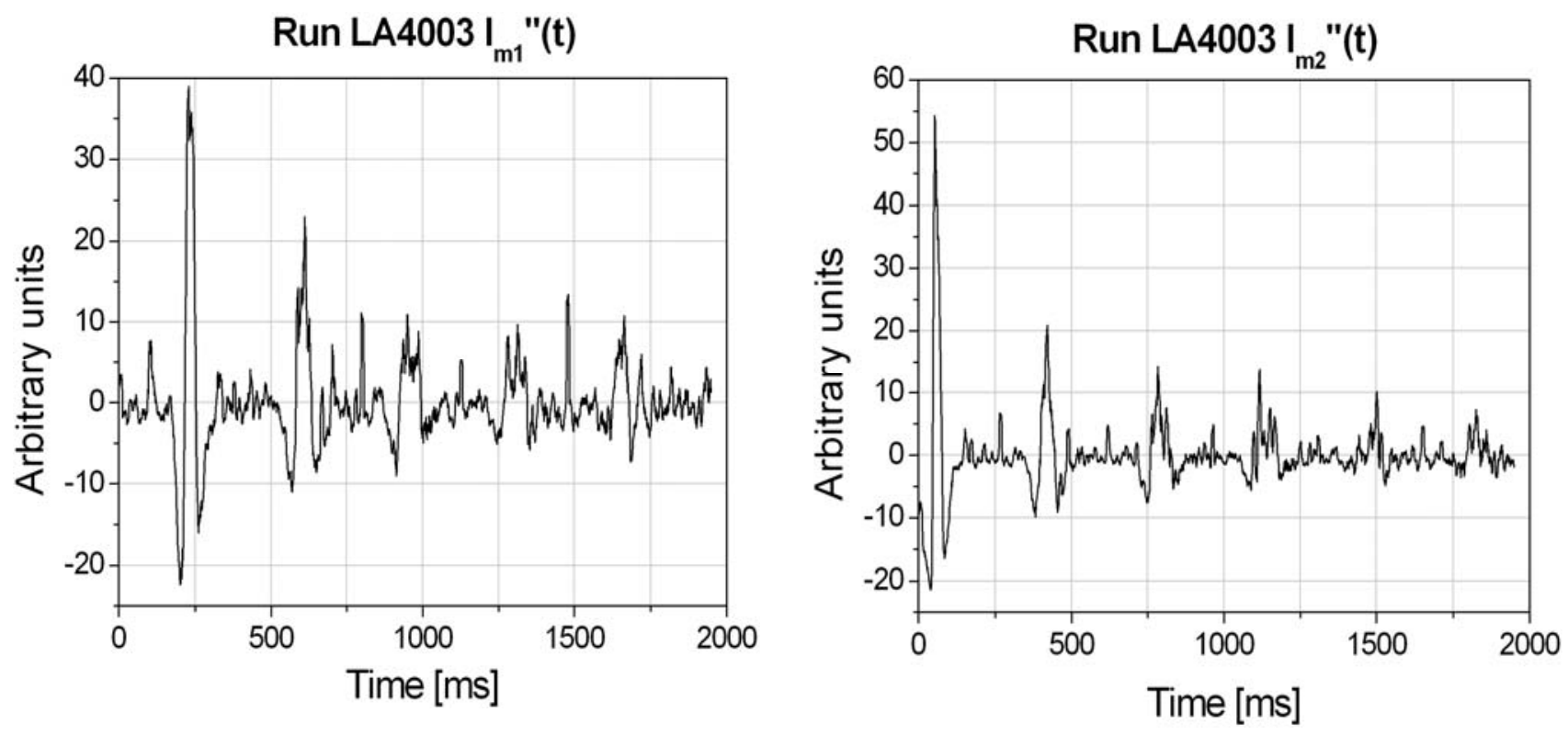

Fig. 8. Signals prepared for correlation calculations in the experiment LA4003.
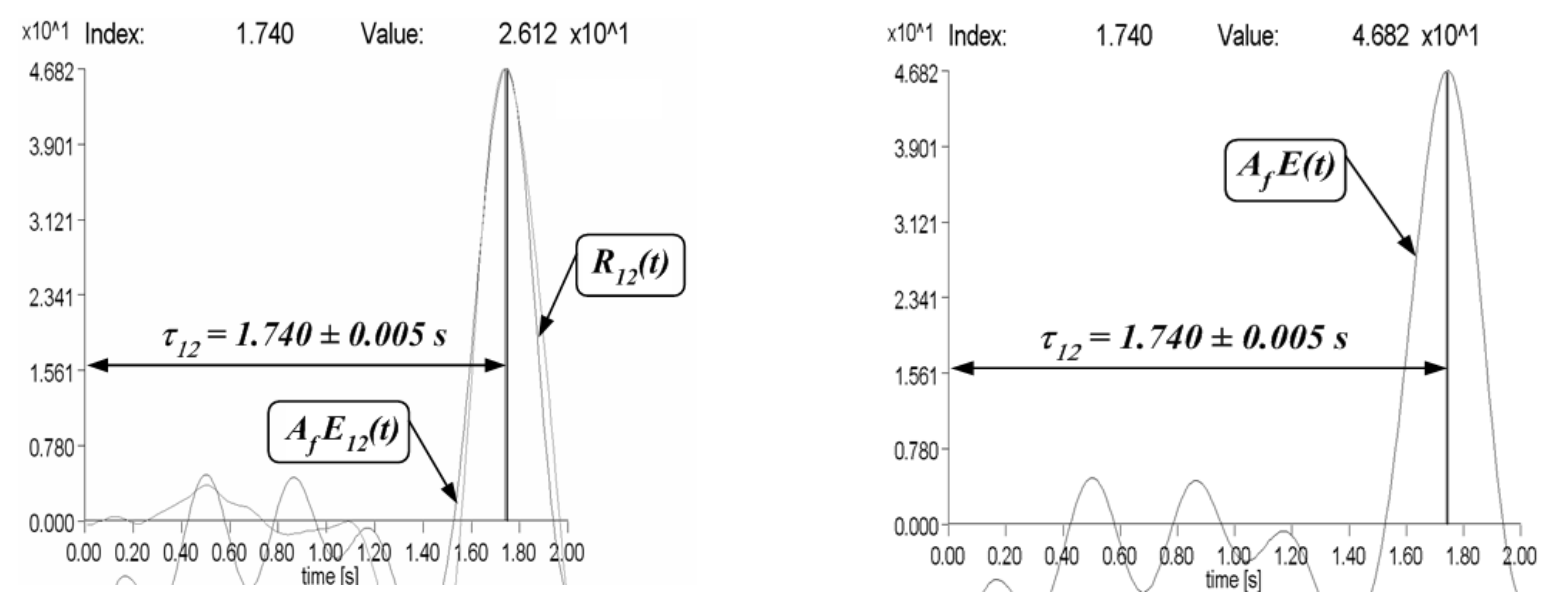

Fig. 9. Comparison of the cross correlation $R_{12}(t)$ with Residence Time Distribution $E_{12}(t)$.

Table 1. Comparison of the experiment's LA4003 results

\begin{tabular}{|c|c|c|c|}
\hline Method & $\begin{array}{c}\boldsymbol{\tau}_{12} \\
{[\mathrm{~s}]}\end{array}$ & $\begin{array}{c}\boldsymbol{v} \\
{[\mathrm{m} / \mathrm{s}]}\end{array}$ & $\begin{array}{c}\boldsymbol{\sigma} \\
{[\%]}\end{array}$ \\
\hline Moments & $1.74 \pm 0.14$ & $11.6 \pm 0.9$ & 8 \\
\hline Deconvolution & $1.740 \pm 0.005$ & $11.69 \pm 0.03$ & 0.3 \\
\hline X-correlation & $1.740 \pm 0.005$ & $11.69 \pm 0.03$ & 0.3 \\
\hline
\end{tabular}

The presented experiment illustrates application of the proposed procedures in comparison to the traditional one. It is easy to recognize how in this case, the propose processing of the same experiment's data, improves 30 times accuracy of the velocity measurement. Moreover, it allows better interpretation of tracer's experiments and extending range of possible flow investigations.

\section{Conclusions}

The presented methods and described experiment, as well as attached literature, allow to formulate the following proposals:

- radioisotopes may profitably supplement traditional method of multiphase flow analysis [15-17],

- reduction of the necessary assumption demanded for radiotracer experiments allows applying easier measuring procedures and extending their range of application [18-25],

- huge development in radiotracer methods, especially in flow velocity measurements, allows both reduction of the radioactivity and increase of the measurements' accuracy [20, 26-29], 
- in many cases, application of radioisotopes may bring smaller hazard due to mass of radioisotopes reduction [30-33],

- in some measurements radiotracers may be replaced by sealed radioactive sources $[10,16,26]$

- equipment based on a pair of sealed radioactive sources appeared to be convenient for two-phase flow control [34-36],

- simultaneous application of tracers and sealed sources gives possibility of more detailed analysis of complex flows [37-40].

\section{Acknowledgements}

All presented investigations were possible due to support under the project P105/10/1574 of the Grant Agency of the Czech Republic, and RVO: 67985874 of the ASCR, as well as the Project No N N523 755340 of the National Science Centre of Poland, which are gratefully acknowledged.

\section{References}

1. C.M. Allen, E.A. Taylor, Trans. Am. Soc. Mech. Eng. 45, 285-341 (1923)

2. J.S. Charlton et al. Guidebook on Radioisotope Tracers in Industry (IAEA Tech. Rep. Ser. 316, Vienna, 1990)

3. C.G. Clayton, J. Brit. Nucl. Eng. Soc. 252-68 (1964)

4. C.W. Sheppard, Principle of the Tracer Method (John Wiley and Sons, NY, 1962)

5. G. Falcone, G.F. Hewitt, C. Alimonti, Multiphase flow metering: principles and applications (Elsevier, Amsterdam, 2009)

6. G. Johansen, P. Jackson, Radioisotope gauges for industrial process measurements (John Wiley and Sons, NY, 2004)

7. V. Mosorov, Appl. Rad. Isotop. 103, 65 (2015)

8. V. Mosorov, M. Zych, R. Hanus, L. Petryka, Appl. Rad. Isotop. 112 136-140 (2016)

9. P.V. Danckwerts, Chem. Eng. Sci. 2, 1 (1953)

10. L. Petryka, R. Keblowski, Precise velocity measurements in two-phase flow by means of sealed radioactive sources. Report of Contract 9326 to IAEA 1-28 (1999)

11. L. Petryka, Proc. of Int. Conf. on Transport and Sedimentation of Solid Particles, Prague Jan. 24-26, E5-1 $\div$ E5-6 (1995)

12. J.G. Proakis, D.G. Manolakis, Digital Signal Processing (Macmillan Publishing Company, N. Y. 1992)

13. L. Petryka, J. Oszajec, Nucl. Geoph. 7, 323-333 (1993)

14. L. Petryka, IAEA Consultant's Meeting on "Radiometric cross-correlation techniques for flow rate measurement”, Krakow, Poland, 1 - 31, (2001)

15. B.K. Arvoh, R. Hoffmann, M. Halstensen, Flow. Meas. Instrum. 23, 56-65 (2012)

16. L. Petryka, et al, EPJ WoC 92, 02065 (2015)
17. M. Kutz (ed.), Handbook of Measurement in Science and Engineering (John Wiley and Sons, NY, 2013)

18. J. Sobota, S. Boczarski, L. Petryka, M. Zych, Proc. of OMS-2005, Changsha, Hunan, China, Oct. 9-13, (2005)

19. J.S. Bendat, A.G. Piersol, Random data - analysis and measurement procedures (John Wiley and Sons, NY, 2010)

20. L. Petryka, M. Zych, R. Hanus, J. Sobota, P. Vlasak, Proc. of 10th ISOPE OMS-2013, Szczecin, Poland, Sept. 22-26, 230-233 (2013)

21. L. Petryka, M. Zych, R. Murzyn, Nukleonika 50, 43$46(2005)$

22. R. Hanus, L. Petryka, M. Zych, Flow Meas. Instrum. 40, 58-63 (2014)

23. R. Hanus, M. Zych, L. Petryka, V. Mosorov, P. Hanus, EPJ WoC 92, 02020 (2015)

24. http://www.oilsandsmagazine.com/

25. P. Vlasak, Z. Chara, J. Krupicka, J. Konfrst, J. Hydrol. Hydrom. 62, 241-247 (2014)

26. L. Petryka, Keynote Lecture. Proc. 12th Int. Conf. on $T \& S$, Prague, Czech Republic, Sept. 20-24, Vol. 1, 59-74 (Ed. AR, Wrocław, 2004)

27. O. Dubé, D. Dubé, J. Chaouki, F. Bertrand, Appl. Radiat. Isot. 89, 109 (2014)

28. J.S. Charlton, Radioisotope Techniques for Problem Solving in Industrial Process Plants (Leonard Hill, Glasgow and London, 1986)

29. S.-H. Jung, J.-S. Kim, J.-B. Kim, T.-Y. Kwon, App. Appl. Rad. Isotop. 67 1254-1258 (2009)

30. L. Furman, L. Petryka, Z. Stęgowski, A. Wierzbicki, Nucl. Instr. \& Meth. in Phys. Res. B. 211 (3) 436-442 (2003)

31. J. Max, Methodes et techniques du traitement du signal et application aux mesures physiques, Vol 1, (Paris 1972)

32. F. Mesch, R. Fritsche, H. Kipphan, J. Dyn. Meas. Control. ASME Trans. 96 414-20 (1974)

33. L. Petryka, M. Zych, R. Murzyn, Tracers and tracing methods, Ciechocinek, Poland, June 22-24, 34-39 (2004)

34. K. Przewłocki, L. Furman, L. Petryka, Z. Stęgowski, J. Kamiński, Hydrol. Hydromech. 44 (1) 3-11(1996)

35. G.J. Roach, T.S. Whitaker, M.J. Millen, Duet Multiphase Flow Meter. The APEA Journal 40 Part 1, 492-498 (2000)

36. H. Santos-Cottin, These, Universite Paul Sabatier, Toulouse (1976)

37. J. Sobota, S. Boczarski, L. Petryka, Proc.of 4th ISOPE IOM, Szczecin Poland, 127-131 (2001)

38. J. Thyn, R. Zitny, J. Kluson, T. Cechak, Analysis and diagnostics of industrial processes by radiotracers and radioisotope sealed sources I (CTU Prague, 2000)

39. J. Thyn, R. Zitny, Analysis and diagnostics of industrial processes by radiotracers and radioisotope sealed sources II (CTU Prague, 2002)

40. R. Zitny, Tracers and tracing methods, Ciechocinek, Poland, June 22-24, 52-61 (2004) 